\title{
Nutritional Supplementation Inhibits the Increase in Serum Malondialdehyde in Patients with Wet Age-Related Macular Degeneration
}

\author{
Toshiyuki Matsuura, ${ }^{1}$ Kei Takayama, ${ }^{1}$ Hiroki Kaneko, ${ }^{1}$ Fuxiang Ye, ${ }^{1,2}$ \\ Hiroshi Fukukita, ${ }^{1}$ Taichi Tsunekawa, ${ }^{1}$ Keiko Kataoka, ${ }^{1}$ Shiang-Jyi Hwang, ${ }^{1,3}$ \\ Yosuke Nagasaka, ${ }^{1}$ Yasuki Ito, ${ }^{1}$ and Hiroko Terasaki ${ }^{1}$ \\ ${ }^{1}$ Department of Ophthalmology, Nagoya University Graduate School of Medicine, Nagoya 466-8550, Japan \\ ${ }^{2}$ Department of Ophthalmology, Shanghai First People's Hospital, Shanghai Jiao Tong University School of Medicine, \\ Shanghai 200240, China \\ ${ }^{3}$ Laboratory of Bell Research Center-Department of Obstetrics and Gynecology Collaborative Research, \\ Nagoya University Graduate School of Medicine, Nagoya 466-8550, Japan
}

Correspondence should be addressed to Hiroki Kaneko; h-kaneko@med.nagoya-u.ac.jp

Received 9 October 2016; Revised 4 December 2016; Accepted 21 December 2016; Published 24 January 2017

Academic Editor: Yuhei Nishimura

Copyright ( 2017 Toshiyuki Matsuura et al. This is an open access article distributed under the Creative Commons Attribution License, which permits unrestricted use, distribution, and reproduction in any medium, provided the original work is properly cited.

\begin{abstract}
Purpose. To compare serum levels of malondialdehyde (MDA) in patients with wet age-related macular degeneration (wAMD), patients with dry AMD (dAMD), and patients without AMD and to evaluate the efficacy of nutritional supplementation for treating elevated serum MDA in patients with wAMD. Methods. MDA levels were measured in sera from 20 patients with wAMD, 20 with dAMD, and 24 without AMD. Patients with wAMD were randomized to receive or not receive nutritional supplementation (10 patients in each group), and MDA levels were measured after 3 months of treatment. Results. MDA levels in patients with wAMD were significantly greater compared with patients without AMD. In eyes with wAMD, there was a significant correlation between MDA levels and choroidal neovascularization lesion area. Serum MDA levels decreased in most patients that received supplementation and significantly increased in those who did not. Conclusion. Baseline serum MDA levels were elevated in patients with wAMD, and MDA levels were directly correlated with choroidal neovascularization lesion area. In addition, nutritional supplementation appeared to exert a protective effect against oxidative stress in patients with wAMD.
\end{abstract}

\section{Introduction}

Age-related macular degeneration (AMD) is a leading cause of blindness in developed countries [1,2]. AMD is classified as either wet AMD (wAMD) or dry AMD (dAMD) according to its pathophysiology [3]. wAMD is characterized by choroidal neovascularization (CNV) and an increase in intraretinal/subretinal fluid which is strongly associated with the overexpression of vascular endothelial growth factor (VEGF). dAMD is characterized by the atrophy of retinal pigment epithelium (RPE) and its advanced form is called geographic atrophy $(\mathrm{GA})[4,5]$. Multiple risk factors, including obesity [6], hypertension [7], smoking [8], and light exposure $[9,10]$, have been shown to contribute to the pathogenesis of AMD, presumably by inducing oxidative stress [11-13].

Nutritional supplements, that is, Ocuvite PreserVision with Lutein, have shown to provide therapeutic benefits in both wAMD and dAMD [14-16]. The Age-Related Eye Disease Study 2 (AREDS 2) found that high-dose zinc/antioxidant supplementation inhibited the progression of early stage AMD to late stage AMD compared with placebo [17, 18]. A previous study demonstrated that mean serum levels of cysteine, an oxidative stress marker, decreased in study participants on a regulated diet that received a 5-day course of antioxidant and zinc supplementation [19]. 
Malondialdehyde (MDA) is a highly reactive threecarbon dialdehyde product of polyunsaturated fatty acid (PUFA) peroxidation by free radicals [20, 21]. Consuming high levels of linoleic acid, a $\omega-6$ fatty acid and the most abundant dietary PUFA, is a risk factor for multiple types of cancers [22] and heart diseases [23]. Photoreceptor outer segments are rich in unsaturated fatty acids. As MDA is generated from the oxidation of unsaturated fatty acids, MDA is present in drusen, the extracellular deposits that accumulate in AMD eyes [24, 25]. MDA is used as a biological marker of oxidative stress [26], suggesting that serum MDA levels are likely to be elevated in patients with AMD compared with healthy subjects [27-29]. Consistent with this hypothesis, MDA exerts cytotoxic effects and upregulates VEGF expression in RPE cells in vitro [30, 31]. We previously demonstrated that not only is MDA a marker of AMD but it also induced autophagy dysregulation and VEGF secretion in AMD eyes. Furthermore, higher levels of dietary linoleic acid intake promoted CNV progression in mice with high MDA levels [32]. In this study, we further analyzed the relationship of clinical characteristics and oxidative stress levels in patients with AMD. In addition, we investigated the efficacy of antioxidant nutritional supplements in reducing serum MDA levels.

\section{Materials and Methods}

2.1. Patients. We prepared serum samples from patients with wet AMD (wAMD group), patients with dry AMD (dAMD group), and individuals without AMD (control group). All of the study patients were $>50$ years old and their axial length was $>23.0 \mathrm{~mm}$ and $<26.0 \mathrm{~mm}$. Patients with polypoidal choroidal vasculopathy, retinal angiomatous proliferation, maculopathy with myopic CNV, or CNV based on angioid streaks or patients with axial length $>26.0 \mathrm{~mm}$ or $<23.0 \mathrm{~mm}$ were excluded from the study. The diagnosis of wAMD and dAMD was established on the basis of age (over 50 years), clinical examination, fundus photography, optical coherence tomography, and fluorescein fundus angiography as previously described $[33,34]$. Patients with wAMD in one eye and $\mathrm{dAMD}$ in the other eye were excluded from the study. Control sera were obtained from patients with other ocular diseases, including cataract, glaucoma, retinal detachment, macular hole, and epiretinal membrane. After baseline measurements were obtained, patients in the wAMD group were divided into two groups; patients in the wAMD group were free to choose whether nutritional supplements should be taken. Patients who took the nutritional supplement were referred to as $S(+)$ and those who did not take it were referred to as $S(-)$. The nutritional supplement (Ocuvite PreserVision with Lutein, Bausch \& Lomb, Rochester, New York City, The United States of America), which was commercially available at the time of the study, contains vitamin C (408 mg), vitamin E (241 mg), zinc $(30 \mathrm{mg})$, and lutein $(9 \mathrm{mg})$. The participants received the supplement once daily for 3 months. The study was approved by the Nagoya University Hospital Ethics Review Board (\#2012-0340-3), and written informed consent was obtained from each patient prior to obtaining the first serum samples.
2.2. Best-Corrected Visual Acuity. The best-corrected visual acuity (BCVA) was measured using a standard Japanese visual acuity chart. The decimal BCVA was converted into the logarithm of the minimum angle of resolution ( $\log M A R)$ for the statistical analysis.

\subsection{Fluorescein Angiography Imaging and Evaluation of} Choroidal Neovascular Lesions. Fluorescein angiography (FA) was recorded for all patients in the wAMD group using cSLO (Heidelberg Retina Angiograph, HRA 2, Heidelberg Engineering, Dossenheim, Germany) as previously described [35-37]. To evaluate the area of the CNV lesion, we traced the border of the area of hyperfluorescein in images captured at $5 \mathrm{~min}$ and quantified the pixels using NAVIS bundled software (Nidek Co. Ltd., Aichi, Japan). The measurements were conducted by two observers (Toshiyuki Matsuura and Kei Takayama) and both observers were blinded to the patients' clinical features. The area of one pixel in the FA images was defined as $0.0004 \mathrm{~mm}^{2}$, and the measurements were converted from pixels to the area $\left(\mathrm{mm}^{2}\right)$.

2.4. Fundus Autofluorescence Imaging and Evaluation of Geographic Atrophy (GA). Fundus autofluorescence (FAF) was recorded using a cSLO (Heidelberg Retina Angiograph, HRA 2, Heidelberg Engineering, Dossenheim, Germany) as previously described [38, 39]. An optically pumped solidstate laser was used to generate the excitation, and emitted light with a wavelength $>500 \mathrm{~nm}$ was detected using a barrier filter. The images were immediately digitized. Then, they were processed using a flexible frame processor and displayed on a computer screen. The FAF images were recorded in accordance with a standard operating procedure. The redfree reflection mode was used for focusing, and a series of $30 \times 30^{\circ}$ images were acquired. To evaluate the area of GA from the FAF images, we traced the border of the dark area in the image and measured the pixels using NAVIS bundled software (Nidek Co. Ltd., Aichi, Japan). The measurements were conducted by two observers (Toshiyuki Matsuura and Kei Takayama) who were blinded to the patients' clinical features. The area of one pixel in the FAF images was $0.0004 \mathrm{~mm}^{2}$, and the measurements were converted from pixels to the area $\left(\mathrm{mm}^{2}\right)$.

2.5. MDA Levels. MDA levels in patient sera were measured at baseline. Then, patients in the wAMD group were randomized to the $S(+)$ group or $S(-)$ group, and sera that were obtained were measured 3 months later. Serum levels of MDA were measured using an OxiSelect MDA Adduct ELISA Kit (STA-332; Cell Biolabs, San Diego, CA, USA) as previously described $[32,40]$. Duplicate evaluations were performed for each sample.

2.6. Statistical Analysis. All of the data are presented as the mean \pm the standard error of the mean. The Mann-Whitney $U$ test was used to compare data between the wAMD, dAMD, and control groups and to compare MDA levels at baseline and after 3 months. Spearman's correlation was used to detect the correlation between MDA levels and age, logMAR BCVA, 
TABLE 1: Patient characteristics in wAMD, dAMD, and control groups.

\begin{tabular}{lccc}
\hline & wAMD & dAMD & Control \\
\hline Number & 20 & 20 & 24 \\
Male/female & $10 / 10$ & $13 / 7$ & $11 / 13$ \\
Mean age (years) & $71.8 \pm 11.0$ & $70.6 \pm 12.5$ & $68.2 \pm 10.2$ \\
Mean logMAR BCVA & $0.29 \pm 0.21$ & $0.24 \pm 0.24$ & $0.22 \pm 0.29$ \\
Mean MDA level $(\mathrm{pmol} / \mathrm{mL})$ & $9.94 \pm 1.53$ & $9.30 \pm 0.92$ & $9.04 \pm 0.96$ \\
Mean CNV area $\left(\mathrm{mm}^{2}\right)$ & $4.65 \pm 3.66$ & & \\
Mean GA area $\left(\mathrm{mm}^{2}\right)$ & & $2.29 \pm 1.80$ & \\
\hline
\end{tabular}

TABLE 2: Patient characteristic in the $S(+)$ and $S(-)$ groups.

\begin{tabular}{lcc}
\hline & $\mathrm{S}(+)$ & $\mathrm{S}(-)$ \\
\hline Number & 10 & 10 \\
Male/female & $5 / 5$ & $5 / 5$ \\
Mean age (years) & $72.0 \pm 9.7$ & $71.6 \pm 10.1$ \\
Mean logMAR BCVA & $0.30 \pm 0.25$ & $0.28 \pm 0.17$ \\
Mean MDA level $(\mathrm{pmol} / \mathrm{mL})$ & $10.34 \pm 2.03$ & $9.54 \pm 0.70$ \\
Mean CNV area $\left(\mathrm{mm}^{2}\right)$ & $4.70 \pm 4.09$ & $4.62 \pm 3.41$ \\
\hline
\end{tabular}

CNV area, and GA area. $P<0.05$ was considered statistically significant.

\section{Results}

3.1. Patient Characteristics. The study included 20 patients in the wAMD group (10 males, mean age $71.8 \pm 11.0$ years), 20 patients in the dAMD group (13 males, mean age $70.6 \pm$ 12.5 years), and 24 patients in the control group (11 males, mean age $68.2 \pm 10.2$ years). The patient characteristics were presented in Table 1 . The mean logMAR BCVA was $0.29 \pm 0.21$, $0.24 \pm 0.24$, and $0.22 \pm 0.29$ in the wAMD, dAMD, and control groups, respectively. Mean levels of MDA were $9.94 \pm$ $1.53 \mathrm{pmol} / \mathrm{mL}, 9.30 \pm 0.92 \mathrm{pmol} / \mathrm{mL}$, and $9.04 \pm 0.96 \mathrm{pmol} / \mathrm{mL}$ in the wAMD, dAMD, and control groups, respectively. Mean $\mathrm{CNV}$ area in the wAMD group was $4.65 \pm 3.66 \mathrm{~mm}^{2}$, and mean GA area in the dAMD group was $2.29 \pm 1.80 \mathrm{~mm}^{2}$. The patient characteristics of the three groups are presented in Table 2. Individual value of MDA-protein adducts and patient information of $S(+)$ and $S(-)$ group are presented in Table 3. The mean logMAR BCVA was $0.30 \pm 0.25$ and $0.28 \pm 0.17$ in the $S(+)$ and $S(-)$ groups, respectively. Mean levels of $\mathrm{MDA}$ were $10.34 \pm 2.03 \mathrm{pmol} / \mathrm{mL}$ in the $\mathrm{S}(+)$ group and $9.54 \pm 0.70 \mathrm{pmol} / \mathrm{mL}$ in the $\mathrm{S}(-)$ group. The mean area of CNV lesions was $4.70 \pm 4.09 \mathrm{~mm}^{2}$ in the $\mathrm{S}(+)$ group and $4.62 \pm 3.41 \mathrm{~mm}^{2}$ in the $\mathrm{S}(-)$ group. There were no significant differences between the $S(+)$ and $S(-)$ groups in any of the parameters evaluated.

3.2. MDA Level. To investigate the relationship between MDA levels and AMD, we evaluated serum MDA levels at baseline and after 3 months of treatment (Figure 1). We observed a $15.0 \%$ increase in mean serum MDA in the wAMD group $(9.94 \pm 1.53 \mathrm{pmol} / \mathrm{mL})$ compared with the control group $(9.04 \pm 0.96 \mathrm{pmol} / \mathrm{mL}, P=0.031)$. An increase in MDA was also observed in the dAMD group $(9.30 \pm 0.92 \mathrm{pmol} / \mathrm{mL})$ compared with the control group, although the difference was not significant $(P=0.12$; Figure $1(\mathrm{a}))$.

Mean levels of MDA in the $S(+)$ group decreased from $10.34 \pm 2.03 \mathrm{pmol} / \mathrm{mL}$ at baseline to $8.88 \pm 1.18 \mathrm{pmol} / \mathrm{mL}$ after 3 months of supplementation $(P=0.064)$. Mean levels of MDA in the S (-) group significantly increased from $9.54 \pm$ $0.70 \mathrm{pmol} / \mathrm{mL}$ at baseline to $10.41 \pm 1.36 \mathrm{pmol} / \mathrm{mL}$ after 3 months $(P=0.012)$ (Figure 1(b)). MDA levels decreased in 7 of the 10 patients in the $S(+)$ group and increased in the other 3 patients (Figure 1(c)). In contrast, MDA levels decreased in 2 of the 10 patients in the $S(-)$ group and increased in 8 patients (Figure 1(d)).

3.3. Correlation between Serum MDA Levels and Patient Characteristics. Next, we examined the correlation between baseline MDA levels and age, logMAR BCVA, CNV area, and $\mathrm{GA}$ area. Representative images of $\mathrm{CNV}$ in eyes with wAMD and GA area in eyes with dAMD are shown in Figure 2. In the representative eye with wAMD, $\log M A R$ BCVA was 0.30 , baseline serum MDA was $10.28 \mathrm{pmol} / \mathrm{mL}$, and CNV area was $3.27 \mathrm{~mm}^{2}$ (Figures 2(a) and 2(b)). In the representative eye with dAMD, logMAR BCVA was 0.097, serum MDA level was $8.90 \mathrm{pmol} / \mathrm{mL}$, and $\mathrm{CNV}$ area was $3.40 \mathrm{~mm}^{2}$ (Figures 2(c) and $2(\mathrm{~d}))$.

In the wAMD group, there was a significant correlation between baseline MDA levels and CNV area $(P=0.038$, $r=0.39$, Figure $3(\mathrm{c}))$, although we did not detect significant correlations between MDA levels and age $(P=0.31$, Figure $3(\mathrm{a}))$ or $\operatorname{logMAR}$ BCVA $(P=0.13$, Figure $3(\mathrm{~b}))$. In the dAMD group, baseline MDA levels were not significantly correlated with age, logMAR BCVA, or GA area $(P=0.33$, 0.14 , and 0.052 , resp.) (Figures 3(d) $-3(\mathrm{f})$ ).

\section{Discussion}

The association between AMD and oxidative stress both in vitro and in vivo has been demonstrated in several studies $[12,41,42]$. We previously demonstrated that MDA is not only a marker of AMD but also a direct contributor to the pathogenesis of AMD [32]. Prospective studies demonstrated that serum markers of oxidative stress are low in patients taking various nutritional supplements, including antioxidants such as lutein, vitamin $\mathrm{C}$, vitamin $\mathrm{E}, \beta$-carotene, and zinc oxide. However, only one study evaluated a 5 -day course of nutritional supplementation and included a relatively small number of patients [19]. In the present study, we measured MDA levels in the sera using ELISA and regarded them as a systemic oxidative stress marker because a previous report describing the complements binds MDA accumulating in the drusen of the eyes with AMD and complement factor $\mathrm{H}$ genetically plays an important role in this complement-MDA cleavage [25]. We were able to demonstrate the usefulness of supplementation by measuring MDA-protein adducts in this study. However, free MDA-protein levels measured by high performance liquid chromatography or 4-hydroxy-2nonenal are considered as more reliable biomarkers of lipid peroxidation in the human plasma [26, 43]. More reliable correlations could be estimated by measuring them. In the 
TABLE 3: Patients of S (+) and S (-) group.

\begin{tabular}{|c|c|c|c|c|c|c|}
\hline \# & Age (years) & Sex & logMAR BCVA & $\mathrm{CNV}$ area $\left(\mathrm{mm}^{2}\right)$ & Before MDA $(\mathrm{pmol} / \mathrm{mL})$ & After MDA (pmol/mL) \\
\hline \multicolumn{7}{|c|}{$\mathrm{S}(+)$ group } \\
\hline 1 & 81 & M & 0.70 & 9.09 & 8.94 & 7.41 \\
\hline 2 & 81 & $\mathrm{~F}$ & 0.70 & 6.70 & 8.45 & 10.23 \\
\hline 3 & 80 & $\mathrm{M}$ & 0.30 & 1.51 & 8.45 & 9.46 \\
\hline 4 & 74 & $\mathrm{M}$ & 0.00 & 11.41 & 10.74 & 9.46 \\
\hline 5 & 74 & M & 0.10 & 7.37 & 9.71 & 7.68 \\
\hline 6 & 73 & $\mathrm{~F}$ & 0.40 & 1.45 & 9.52 & 8.90 \\
\hline 7 & 72 & $\mathrm{~F}$ & 0.22 & 0.85 & 11.07 & 9.46 \\
\hline 8 & 69 & M & 0.30 & 7.27 & 14.28 & 8.86 \\
\hline 9 & 68 & $\mathrm{~F}$ & 0.30 & 1.01 & 8.94 & 10.23 \\
\hline 10 & 50 & $\mathrm{~F}$ & 0.00 & 0.30 & 13.29 & 6.91 \\
\hline \multicolumn{7}{|c|}{$\mathrm{S}(-)$ group } \\
\hline 1 & 94 & $\mathrm{~F}$ & 0.30 & 0.85 & 9.71 & 9.98 \\
\hline 2 & 81 & $\mathrm{~F}$ & 0.30 & 6.61 & 8.69 & 8.51 \\
\hline 3 & 80 & M & 0.40 & 10.69 & 8.90 & 9.54 \\
\hline 4 & 78 & $\mathrm{M}$ & 0.10 & 1.73 & 9.71 & 10.41 \\
\hline 5 & 74 & $\mathrm{M}$ & 0.22 & 5.68 & 8.32 & 9.54 \\
\hline 6 & 74 & $\mathrm{~F}$ & 0.70 & 5.53 & 9.71 & 10.74 \\
\hline 7 & 67 & $\mathrm{~F}$ & 0.10 & 1.25 & 10.41 & 12.17 \\
\hline 8 & 59 & $\mathrm{~F}$ & 0.22 & 8.53 & 9.71 & 13.30 \\
\hline 9 & 55 & $\mathrm{M}$ & 0.22 & 1.12 & 9.71 & 10.23 \\
\hline 10 & 54 & $\mathrm{M}$ & 0.22 & 4.18 & 10.48 & 9.72 \\
\hline
\end{tabular}

present study, we demonstrated that mean serum MDA levels decreased in patients on a regulated diet that received nutritional supplementation, Ocuvite PreserVision with Lutein, for three months, whereas mean levels increased in patients on a regulated diet with no nutritional supplementation. As systemic oxidative stress is a key contributor to the pathogenesis of AMD, these findings suggest that nutritional supplementation might have inhibited systemic oxidative stress in these patients.

In patients with wAMD, MDA levels were significantly correlated with CNV area (Figure 3(c)). CNV is defined as neovascularization resulting from oxidative stress-induced damage to the choroid and RPE [44-46]. We previously demonstrated that MDA levels in the RPE and choroid of AMD patients were significantly elevated compared with control subjects and that MDA administration induced the upregulation of VEGF expression in RPE cells and induced an increase in CNV volume in vivo [32], suggesting that serum MDA levels are correlated with CNV area. To measure CNV lesion area, FA/ICGA were performed in the wAMD group, as previously described. Changes in the CNV area in the $S(+)$ and $S(-)$ groups would be detectable by FA/ICGA after 3-month observation, although FA/ICGA was not performed in the present study. From OCT images, the CNV area was considered to show no remarkable change after 3-month observation. Therefore, the relation between CNV area expansion and MDA change could not be compared. We did not detect a correlation between MDA and GA area in the dAMD group (Figure 3(f)). GA is defined as a loss in RPE area $[1,47]$. The RPE maintains the health of the retina by providing protection from oxidation $[48,49]$. GA can take more than 6 years to develop, and it arises as a consequence of long-tern exposure to stress [33], rather than current levels of stress. These findings are consistent with the lack of a correlation between GA area and serum levels of MDA in this study. Although we measured only MDA-protein adducts in this study, measuring one more biomarker, for example, 4$\mathrm{HNE}$, is preferable in the further study.

There are several limitations of the study worth noting. First, the present study included small number of patients and the observational period was only 3 months. More patients and long-term studies are needed to confirm our findings. In addition, the control group should also be treated by the same nutritional supplementation to have a proper control. A better method would be that the patients in the control groups take same supplementation after 3 months and it should be confirmed that their MDA levels will be also reduced after taking supplementation. The present study showed only "tendency $(P=0.068)$," not "significant difference $(P<$ 0.05)," of supplementation for reducing MDA levels after 3 months. It is possible that a longer observation period may result in "significant difference" in MDA levels between S $(+)$ and S (-) groups. However, other factors, that is, food or stress, could affect systemic oxidative stress during the long-time observation. Second, it was not elucidated whether nutritional supplementation directly reduces MDA level in the eye. We previously reported that high levels of MDA were observed in human RPE/choroid obtained from donor eyes from several Eye Banks. The AREDS demonstrated the efficacy of supplementation in the prevention of AMD 


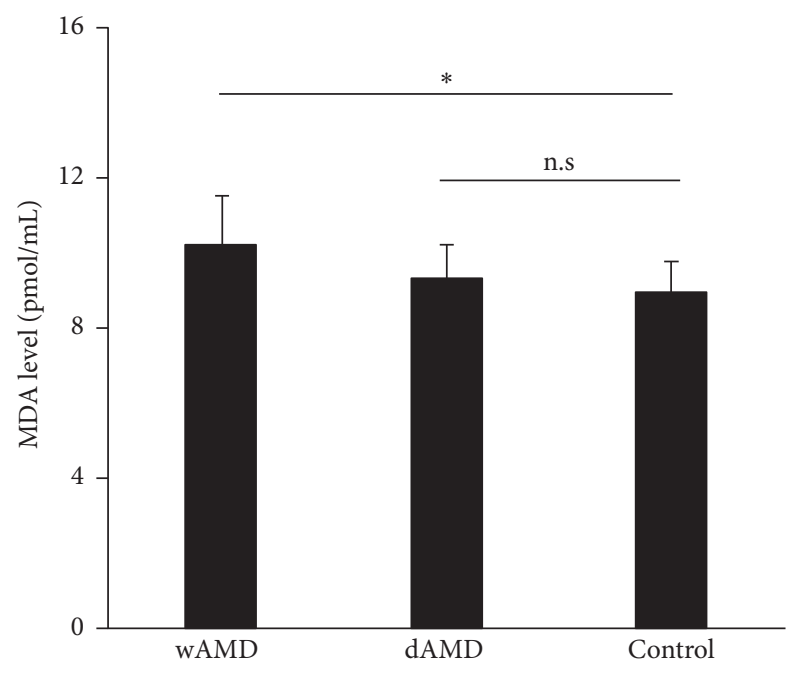

(a)

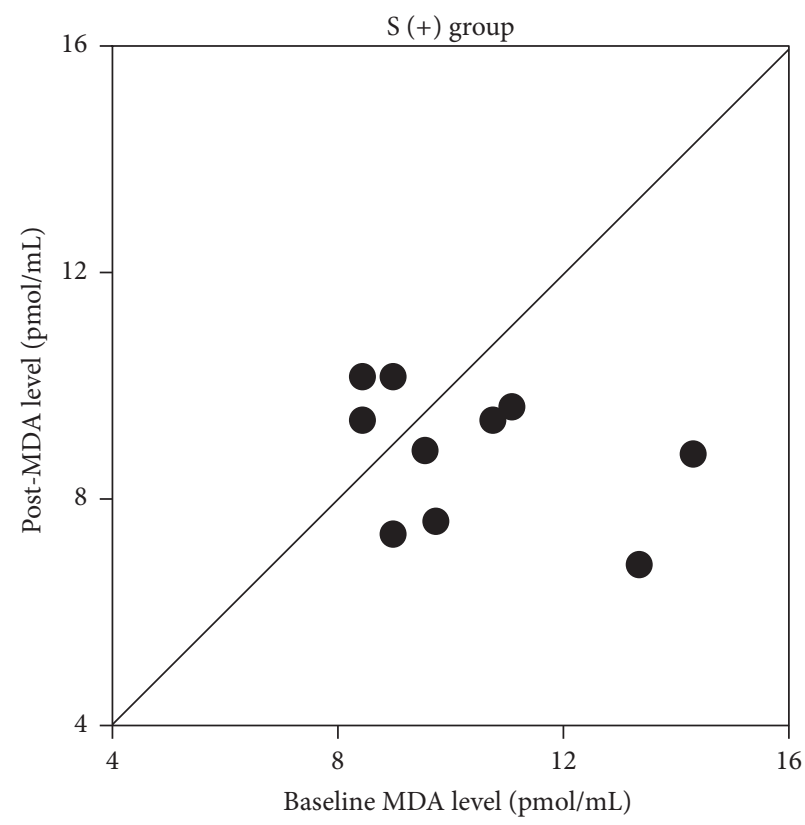

(c)

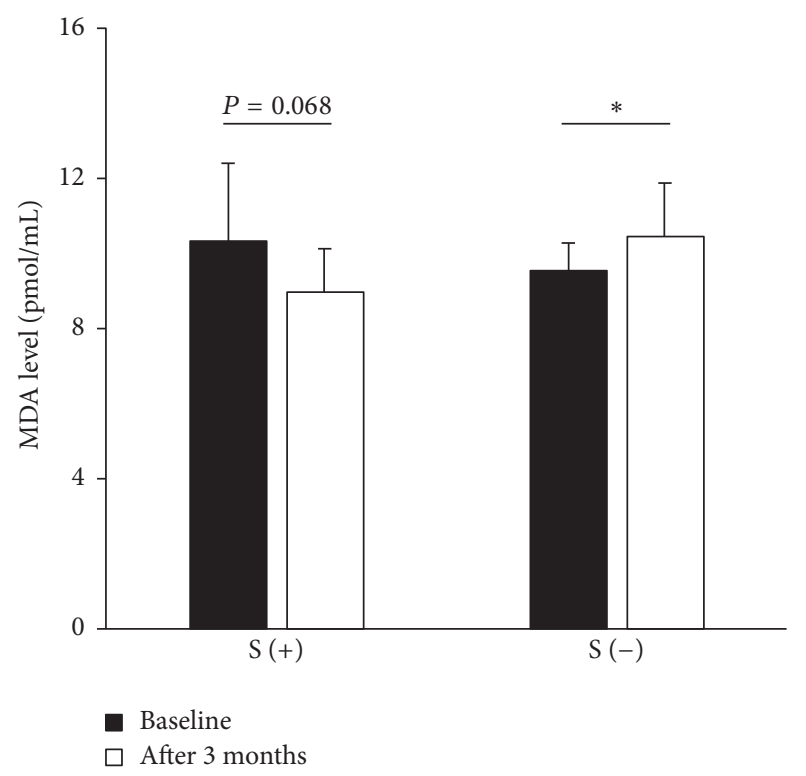

(b)

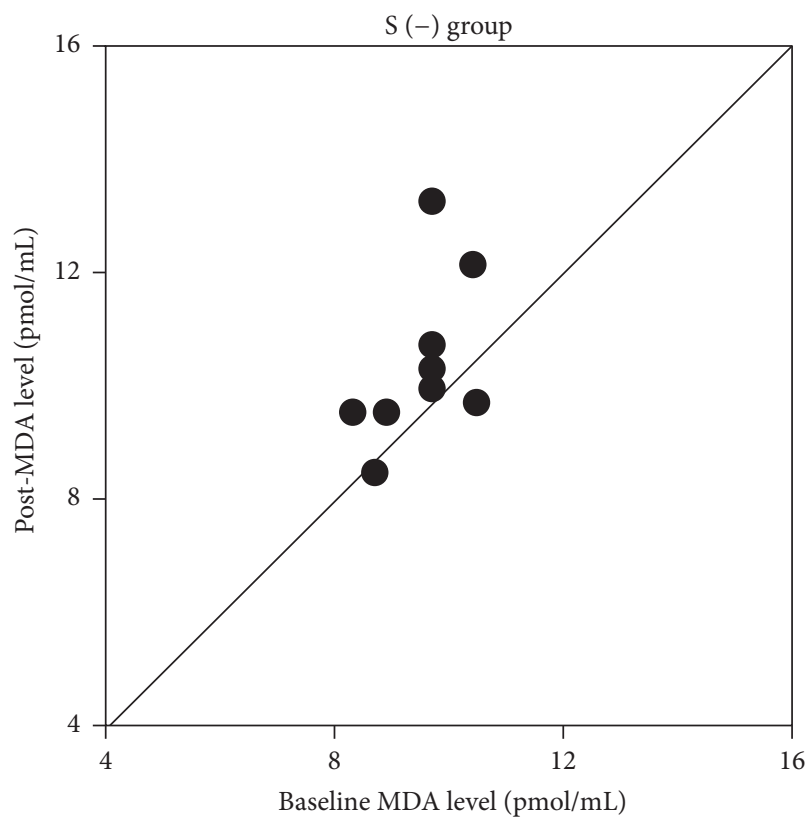

(d)

FIGURE 1: Serum MDA levels in the study population and changes in MDA levels in wAMD patients that received nutritional supplementation. (a) Higher serum MDA levels were observed in the wAMD group. (b) MDA levels tended to decrease in wAMD patients that received supplementation for 3 months. (c) MDA levels decreased in 7 of the 10 patients in the $S(+)$ group and increased in the remaining 3 patients. (d) MDA levels decreased in 2 of the 10 patients in the $S(-)$ and increased in the other 8 patients. ${ }^{*} P<0.05$.

progression over a period of 5 years $[17,50]$. It is difficult to prospectively study the effect of nutritional supplementation on MDA levels in human eyes.

In conclusion, we demonstrated that elevated serum MDA levels were directly associated with the area of CNV lesions in eyes with wAMD and that nutritional supplements appear to protect the eyes from systemic oxidative damage. MDA might be a valuable marker of oxidative stress. The present study is a retrospective short-observation study including small number of patients. To obtain more reliable value of supplementation for AMD and systemic oxidative stress, a double-blinded prospective randomized study of supplementation is necessary.

\section{Competing Interests}

The authors declare that there is no conflict of interests regarding the publication of this paper. 


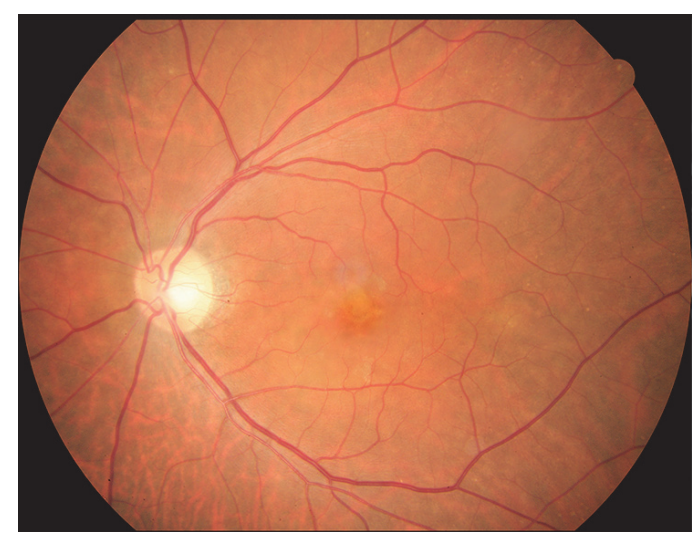

(a)

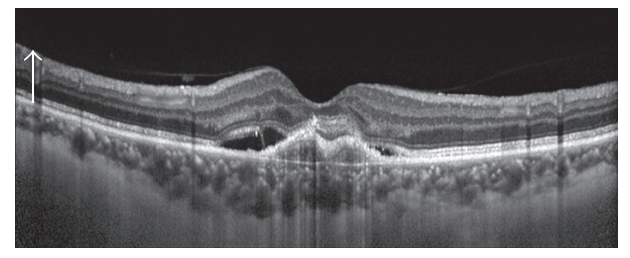

(c)

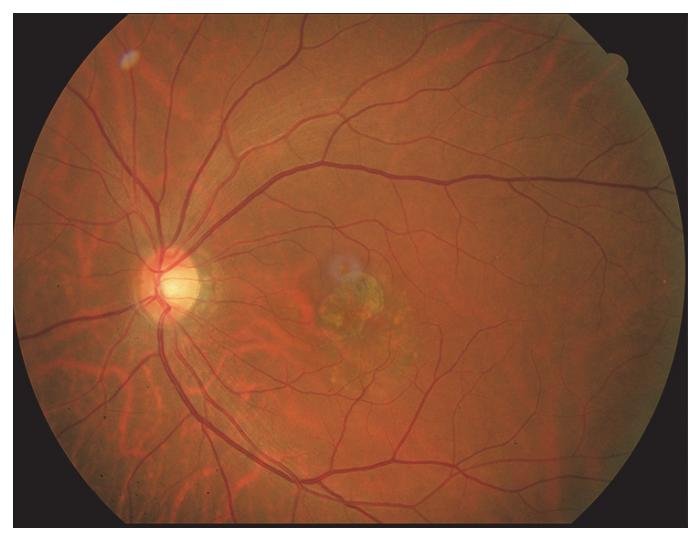

(e)

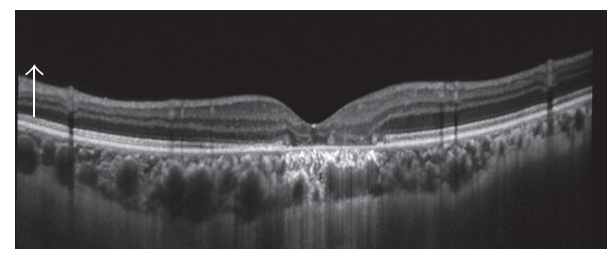

(g)

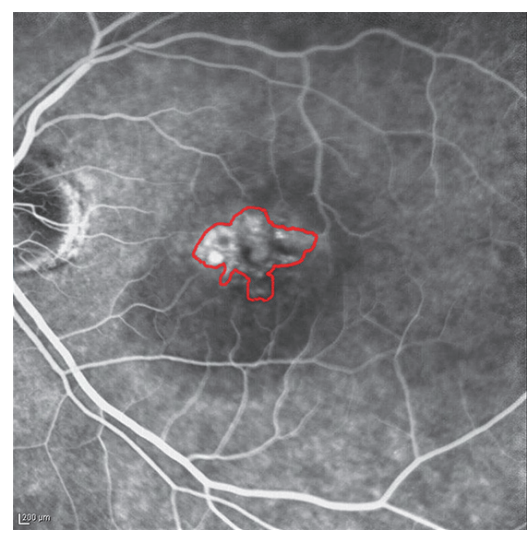

(b)

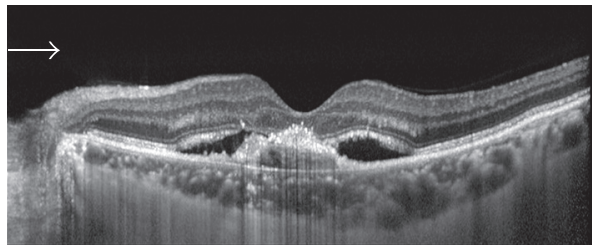

(d)

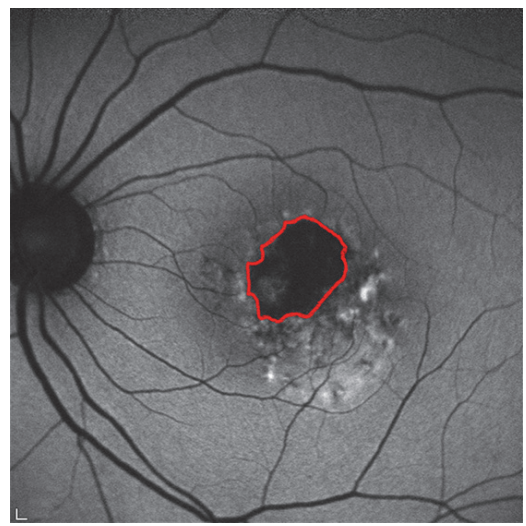

(f)

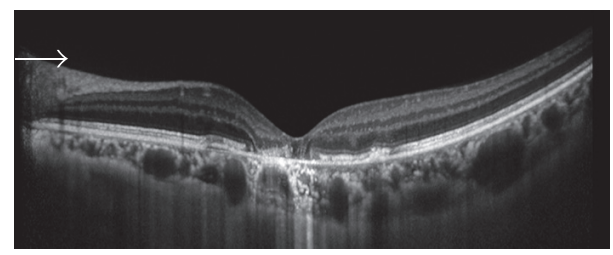

(h)

Figure 2: Representative images of wet AMD and dry AMD. (a, b) The color (a) and fluorescein angiography (FA, b) images of a representative case of wet AMD. The CNV lesion area in FA images was measured. (c, d) Representative case of dry AMD. Geographic atrophy area in the autofluorescein angiography image (d) was measured. (e, f) images are the OCT images of wet AMD and (g, h) are of dry AMD.

\section{Authors' Contributions}

Toshiyuki Matsuura and Kei Takayama contributed equally.

\section{Acknowledgments}

The authors give heartfelt thanks to all the patients who participated in this study. The authors also thank Reona
Kimoto, Chisato Ishizuka, Keigo Ohmori, and Kumi Fujiwara for technical assistance. This work was partially supported by Grants-in-Aid for Scientific Research B (Hiroko Terasaki; 15H04994), Chukyo Longevity Medical and Promotion Foundation (Hiroki Kaneko), Takeda Medical Research Foundation (Hiroki Kaneko), and Takeda Science Foundation (Hiroki Kaneko). Mishima Saiichi-kinen Gankakenkyu 


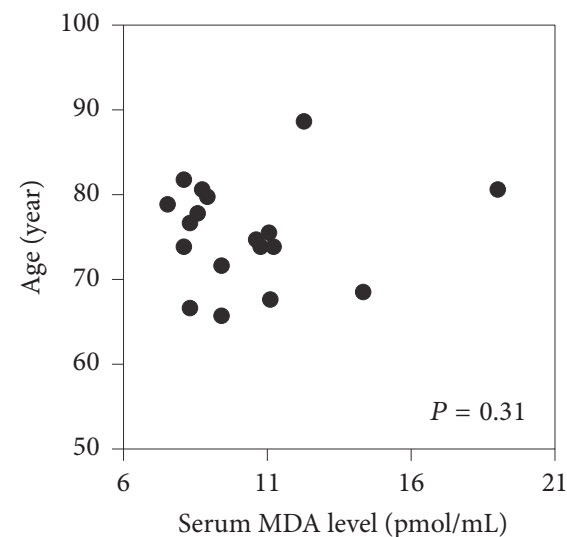

(a)

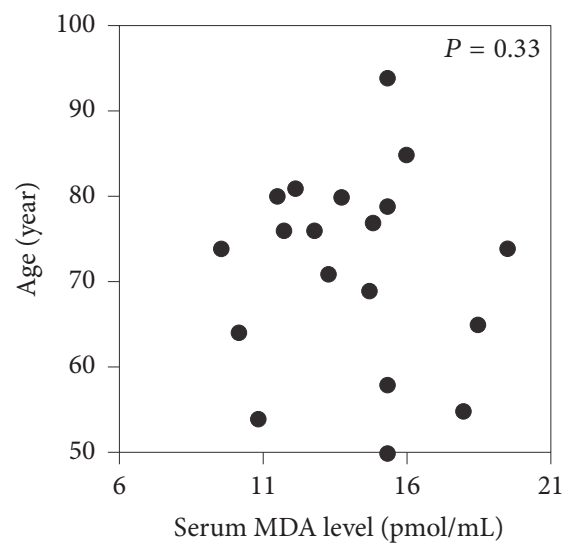

(d)

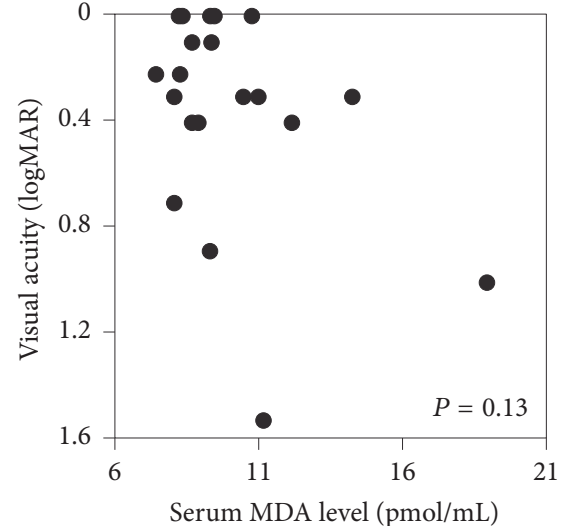

(b)

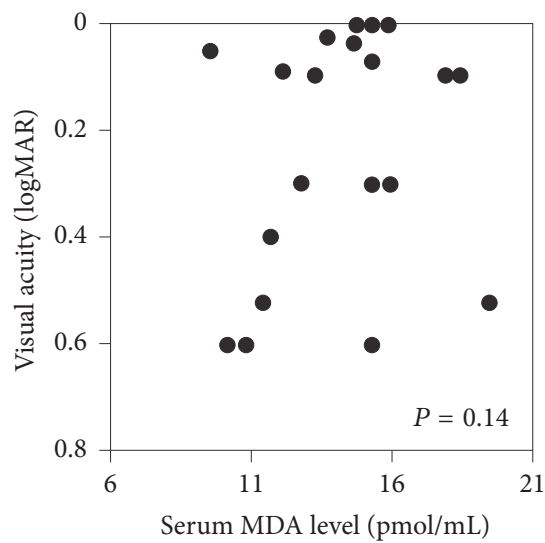

(e)

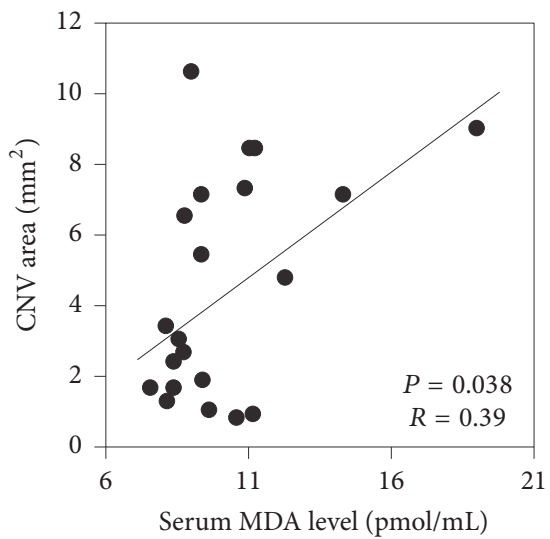

(c)

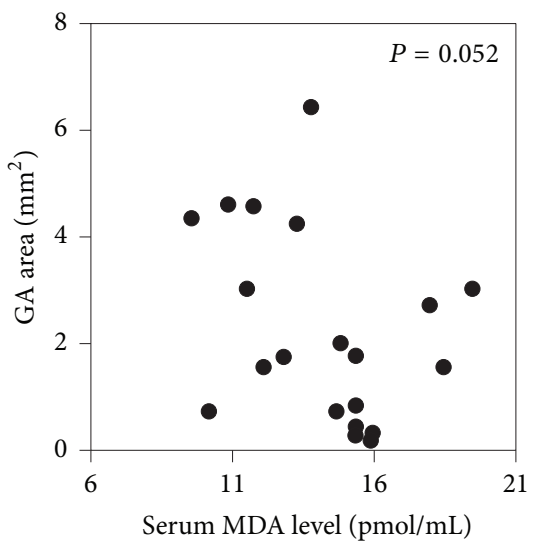

(f)

FIGURE 3: Correlation between MDA levels and patient characteristics in the wet AMD and dry AMD groups. (a-c) In the wet AMD group, serum MDA levels and choroidal neovascularization $(\mathrm{CNV})$ lesion area were significantly correlated, but MDA levels were not correlated with ages or visual acuity. (d-f) In the dry AMD group, there was no significant correlation between serum MDA levels and age, visual acuity, or geographic atrophy (GA) area.

Kokusaikouryu Kikin (Hiroki Kaneko) and Itoh-Chubei Foundation (Hiroki Kaneko) are also acknowledged.

\section{References}

[1] J. Ambati, B. K. Ambati, S. H. Yoo, S. Ianchulev, and A. P. Adamis, "Age-related macular degeneration: etiology, pathogenesis, and therapeutic strategies," Survey of Ophthalmology, vol. 48, no. 3, pp. 257-293, 2003.

[2] A. C. Bird, "Therapeutic targets in age-related macular disease," The Journal of Clinical Investigation, vol. 120, no. 9, pp. 30333041, 2010.

[3] J. Ambati and B. J. Fowler, "Mechanisms of age-related macular degeneration," Neuron, vol. 75, no. 1, pp. 26-39, 2012.

[4] H. Kaneko, S. Dridi, V. Tarallo et al., "DICER1 deficit induces Alu RNA toxicity in age-related macular degeneration," Nature, vol. 471, no. 7338, pp. 325-332, 2011.

[5] P. T. V. M. de Jong, "Age-related macular degeneration," The New England Journal of Medicine, vol. 355, no. 14, pp. 1474-1485, 2006.

[6] Q.-Y. Zhang, L.-J. Tie, S.-S. Wu et al., "Overweight, obesity, and risk of age-related macular degeneration," Investigative Ophthalmology and Visual Science, vol. 57, no. 3, pp. 1276-1283, 2016.
[7] A. Cougnard-Grégoire, M.-N. Delyfer, J.-F. Korobelnik et al., "Long-term blood pressure and age-related macular degeneration: the ALIENOR study," Investigative Ophthalmology and Visual Science, vol. 54, no. 3, pp. 1905-1912, 2013.

[8] N. Joachim, P. Mitchell, G. Burlutsky, A. Kifley, and J. J. Wang, "The incidence and progression of age-related macular degeneration over 15 years: The Blue Mountains Eye Study," Ophthalmology, vol. 122, no. 12, pp. 2482-2489, 2015.

[9] K. Takayama, H. Kaneko, K. Kataoka et al., "Nuclear factor (erythroid-derived)-related factor 2-associated retinal pigment epithelial cell protection under blue light-induced oxidative stress," Oxidative Medicine and Cellular Longevity, vol. 2016, Article ID 8694641, 9 pages, 2016.

[10] T. Schick, L. Ersoy, Y. T. E. Lechanteur et al., "History of sunlight exposure is a risk factor for age-related macular degeneration," Retina, vol. 36, no. 4, pp. 787-790, 2016.

[11] K. M. Bertram, C. J. Baglole, R. P. Phipps, and R. T. Libby, "Molecular regulation of cigarette smoke induced-oxidative stress in human retinal pigment epithelial cells: Implications for age-related macular degeneration," American Journal of Physiology-Cell Physiology, vol. 297, no. 5, pp. C1200-C1210, 2009. 
[12] H. Du, X. Xiao, T. Stiles, C. Douglas, D. Ho, and P. X. Shaw, "Novel mechanistic interplay between products of oxidative stress and components of the complement system in AMD pathogenesis," Open Journal of Ophthalmology, vol. 6, no. 1, pp. 43-50, 2016.

[13] R. A. Armstrong and M. Mousavi, "Overview of risk factors for age-related macular degeneration (AMD)," Journal of Stem Cells, vol. 10, no. 3, pp. 171-191, 2015.

[14] R. Liu, T. Wang, B. Zhang et al., "Lutein and zeaxanthin supplementation and association with visual function in agerelated macular degeneration," Investigative Ophthalmology \& Visual Science, vol. 56, no. 1, pp. 252-258, 2015.

[15] X. Wang, C. Jiang, Y. Zhang, Y. Gong, X. Chen, and M. Zhang, "Role of lutein supplementation in the management of age-related macular degeneration: meta-analysis of randomized controlled trials," Ophthalmic Research, vol. 52, no. 4, pp. 198-205, 2014.

[16] J. Evans, "Antioxidant supplements to prevent or slow down the progression of AMD: a systematic review and meta-analysis," Eye, vol. 22, no. 6, pp. 751-760, 2008.

[17] E. Y. Chew, T. E. Clemons, J. P. SanGiovanni et al., "Secondary analyses of the effects of lutein/zeaxanthin on age-related macular degeneration progression AREDS2 report no. 3," JAMA Ophthalmology, vol. 132, no. 2, pp. 142-149, 2014.

[18] M. E. Aronow and E. Y. Chew, "Age-related eye disease study 2: perspectives, recommendations, and unanswered questions," Current Opinion in Ophthalmology, vol. 25, no. 3, pp. 186-190, 2014.

[19] M. A. Brantley Jr., M. P. Osborn, B. J. Sanders et al., “The shortterm effects of antioxidant and zinc supplements on oxidative stress biomarker levels in plasma: a pilot investigation," American Journal of Ophthalmology, vol. 153, no. 6, pp. 1104-1109, 2012.

[20] Y. F. Njie-Mbye, M. Kulkarni-Chitnis, C. A. Opere, A. Barrett, and S. E. Ohia, "Lipid peroxidation: pathophysiological and pharmacological implications in the eye," Frontiers in Physiology, vol. 4, article no. 366, 2013.

[21] H. Esterbauer, R. J. Schaur, and H. Zollner, "Chemistry and biochemistry of 4-hydroxynonenal, malonaldehyde and related aldehydes," Free Radical Biology and Medicine, vol. 11, no. 1, pp. 81-128, 1991.

[22] H. Okuyama, T. Kobayashi, and S. Watanabe, "Dietary fatty acids-the N-6/N-3 balance and chronic elderly diseases. Excess linoleic acid and relative N-3 deficiency syndrome seen in Japan," Progress in Lipid Research, vol. 35, no. 4, pp. 409-457, 1996.

[23] C. E. Ramsden, D. Zamora, B. Leelarthaepin et al., "Use of dietary linoleic acid for secondary prevention of coronary heart disease and death: evaluation of recovered data from the Sydney Diet Heart Study and updated meta-analysis," British Medical Journal, vol. 346, no. 7894, Article ID e8707, 2013.

[24] F. Schutt, M. Bergmann, F. G. Holz, and J. Kopitz, "Proteins modified by malondialdehyde, 4-hydroxynonenal, or advanced glycation end products in lipofuscin of human retinal pigment epithelium," Investigative Ophthalmology and Visual Science, vol. 44, no. 8, pp. 3663-3668, 2003.

[25] D. Weismann, K. Hartvigsen, N. Lauer et al., "Complement factor $\mathrm{H}$ binds malondialdehyde epitopes and protects from oxidative stress," Nature, vol. 478, no. 7367, pp. 76-81, 2011.

[26] D. Del Rio, A. J. Stewart, and N. Pellegrini, "A review of recent studies on malondialdehyde as toxic molecule and biological marker of oxidative stress," Nutrition, Metabolism \& Cardiovascular Diseases, vol. 15, no. 4, pp. 316-328, 2005.
[27] X. L. Shen, L. H. Jia, P. Zhao et al., "Changes in blood oxidative and antioxidant parameters in a group of chinese patients with age-related macular degeneration," Journal of Nutrition, Health \& Aging, vol. 16, no. 3, pp. 201-204, 2012.

[28] Y. Totan, O. Çekiç, M. Borazan, E. Uz, S. Sögüt, and O. Akyol, "Plasma malondialdehyde and nitric oxide levels in age related macular degeneration," British Journal of Ophthalmology, vol. 85, no. 12, pp. 1426-1428, 2001.

[29] Ö. Yildirim, N. A. Ateş, L. Tamer et al., "Changes in antioxidant enzyme activity and malondialdehyde level in patients with agerelated macular degeneration," Ophthalmologica, vol. 218, no. 3, pp. 202-206, 2004.

[30] M. Bergmann, F. Holz, and J. Kopitz, "Lysosomal stress and lipid peroxidation products induce VEGF-121 and VEGF-165 expression in ARPE-19 cells," Graefe's Archive for Clinical and Experimental Ophthalmology, vol. 249, no. 10, pp. 1477-1483, 2011.

[31] T. U. Krohne, N. K. Stratmann, J. Kopitz, and F. G. Holz, "Effects of lipid peroxidation products on lipofuscinogenesis and autophagy in human retinal pigment epithelial cells," Experimental Eye Research, vol. 90, no. 3, pp. 465-471, 2010.

[32] F. Ye, H. Kaneko, Y. Hayashi et al., "Malondialdehyde induces autophagy dysfunction and VEGF secretion in the retinal pigment epithelium in age-related macular degeneration," Free Radical Biology and Medicine, vol. 94, pp. 121-134, 2016.

[33] H. M. Zajaç-Pytrus, A. Pilecka, A. Turno-Krecicka, J. AdamiecMroczek, and M. Misiuk-Hojło, "The dry form of age-related macular degeneration (AMD): the current concepts of pathogenesis and prospects for treatment," Advances in Clinical and Experimental Medicine, vol. 24, no. 6, pp. 1099-1104, 2015.

[34] M. A. Zarbin, R. P. Casaroli-Marano, and P. J. Rosenfeld, "Agerelated macular degeneration: clinical findings, histopathology and imaging techniques," Developments in Ophthalmology, vol. 53, pp. 1-32, 2014.

[35] K. Takayama, H. Kaneko, S. Ueno et al., "Evaluation of shortterm outcomes of intravitreal aflibercept injections for age-related macular degeneration using focal macular electroretinography," Retina, In press.

[36] K. Takayama, H. Kaneko, K. Kataoka et al., "Short-term focal macular electroretinogram of eyes treated by aflibercept \& photodynamic therapy for polypoidal choroidal vasculopathy," Graefe's Archive for Clinical and Experimental Ophthalmology, 2016.

[37] K. Takayama, Y. Ito, H. Kaneko et al., "Cross-sectional pupillographic evaluation of relative afferent pupillary defect in agerelated macular degeneration," Medicine, vol. 95, no. 39, Article ID e4978, 2016.

[38] F. G. Holz, J. S. Steinberg, A. Göbel, M. Fleckenstein, and S. Schmitz-Valckenberg, "Fundus autofluorescence imaging in dry AMD: 2014 jules gonin lecture of the retina research foundation," Graefe's Archive for Clinical and Experimental Ophthalmology, vol. 253, no. 1, pp. 7-16, 2015.

[39] S. Schmitz-Valckenberg, F. G. Holz, A. C. Bird, and R. F. Spaide, "Fundus autofluorescence imaging: review and perspectives," Retina, vol. 28, no. 3, pp. 385-409, 2008.

[40] P. Brzek, A. Ksiazek, L. Oldakowski, and M. Konarzewski, "High basal metabolic rate does not elevate oxidative stress during reproduction in laboratory mice," Journal of Experimental Biology, vol. 217, no. 9, pp. 1504-1509, 2014.

[41] S. Beatty, H.-H. Koh, M. Phil, D. Henson, and M. Boulton, "The role of oxidative stress in the pathogenesis of age-related macular degeneration," Survey of Ophthalmology, vol. 45, no. 2, pp. 115-134, 2000. 
[42] M. C. Marazita, A. Dugour, M. D. Marquioni-Ramella, J. M. Figueroa, and A. M. Suburo, "Oxidative stress-induced premature senescence dysregulates VEGF and CFH expression in retinal pigment epithelial cells: implications for age-related macular degeneration," Redox Biology, vol. 7, pp. 78-87, 2016.

[43] C. M. Spickett, "The lipid peroxidation product 4-hydroxy-2nonenal: advances in chemistry and analysis," Redox Biology, vol. 1, no. 1, pp. 145-152, 2013.

[44] Y. Li, X. Liu, T. Zhou et al., "Inhibition of APE1/Ref-1 redox activity rescues human retinal pigment epithelial cells from oxidative stress and reduces choroidal neovascularization," Redox Biology, vol. 2, no. 1, pp. 485-494, 2014.

[45] X. Li, Y. Cai, Y.-S. Wang et al., "Hyperglycaemia exacerbates choroidal neovascularisation in mice via the oxidative stressinduced activation of STAT3 signalling in RPE cells," PLoS ONE, vol. 7, no. 10, Article ID e47600, 2012.

[46] F. Ye, H. Kaneko, Y. Nagasaka et al., "Plasma-activated medium suppresses choroidal neovascularization in mice: a new therapeutic concept for age-related macular degeneration," Scientific Reports, vol. 5, article no. 7705, 2015.

[47] A. C. Bird, R. L. Phillips, and G. S. Hageman, "Geographic atrophy: A histopathological assessment," JAMA Ophthalmology, vol. 132, no. 3, pp. 338-345, 2014.

[48] I. Bhutto and G. Lutty, "Understanding age-related macular degeneration (AMD): relationships between the photoreceptor/retinal pigment epithelium/Bruch's membrane/choriocapillaris complex," Molecular Aspects of Medicine, vol. 33, no. 4, pp. 295-317, 2012.

[49] J. Hanus, C. Anderson, and S. Wang, "RPE necroptosis in response to oxidative stress and in AMD," Ageing Research Reviews, vol. 24, pp. 286-298, 2015.

[50] D. E. Bonds, M. Harrington, B. B. Worrall et al., "Effect of longchain $\omega-3$ fatty acids and lutein + zeaxanthin supplements on cardiovascular outcomes results of the age-related eye disease study 2 (AREDS2) randomized clinical trial," JAMA Internal Medicine, vol. 174, no. 5, pp. 763-771, 2014. 


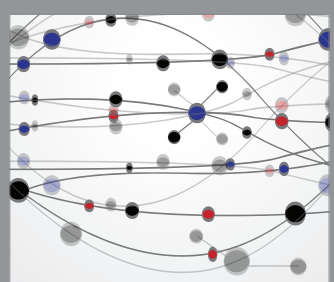

The Scientific World Journal
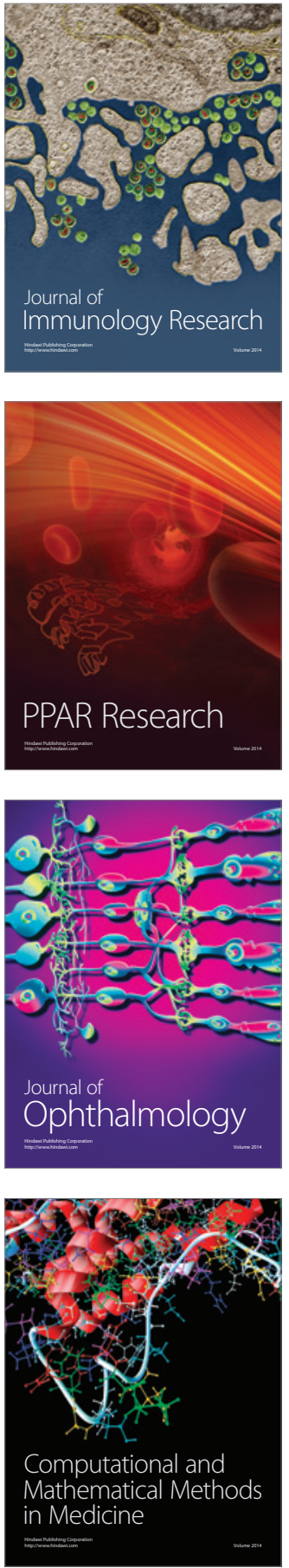

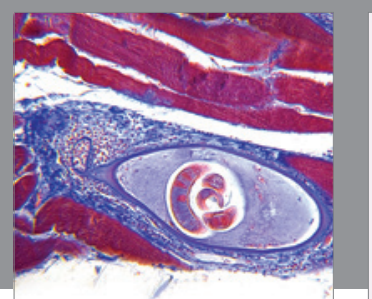

Gastroenterology Research and Practice
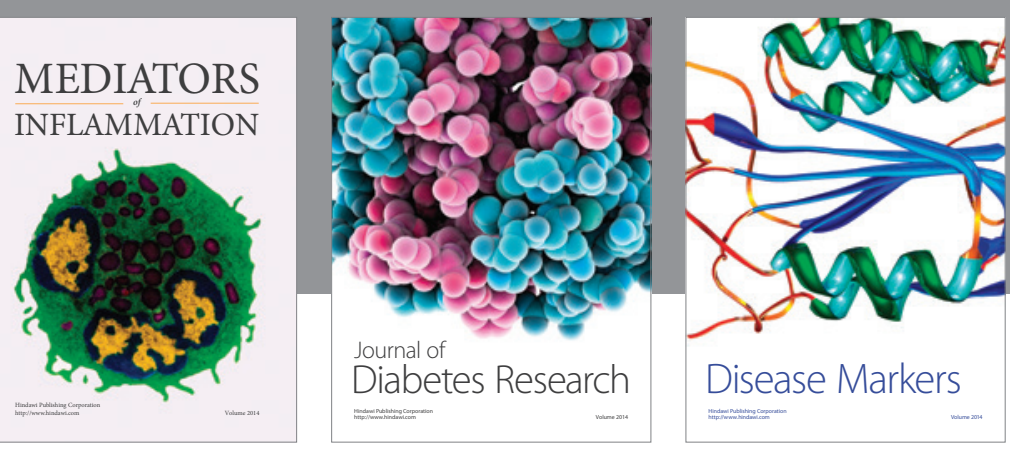

Disease Markers

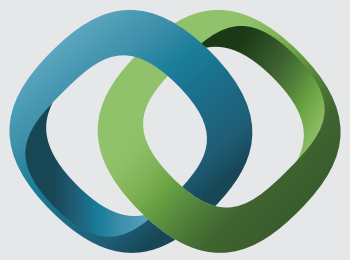

\section{Hindawi}

Submit your manuscripts at

https://www.hindawi.com
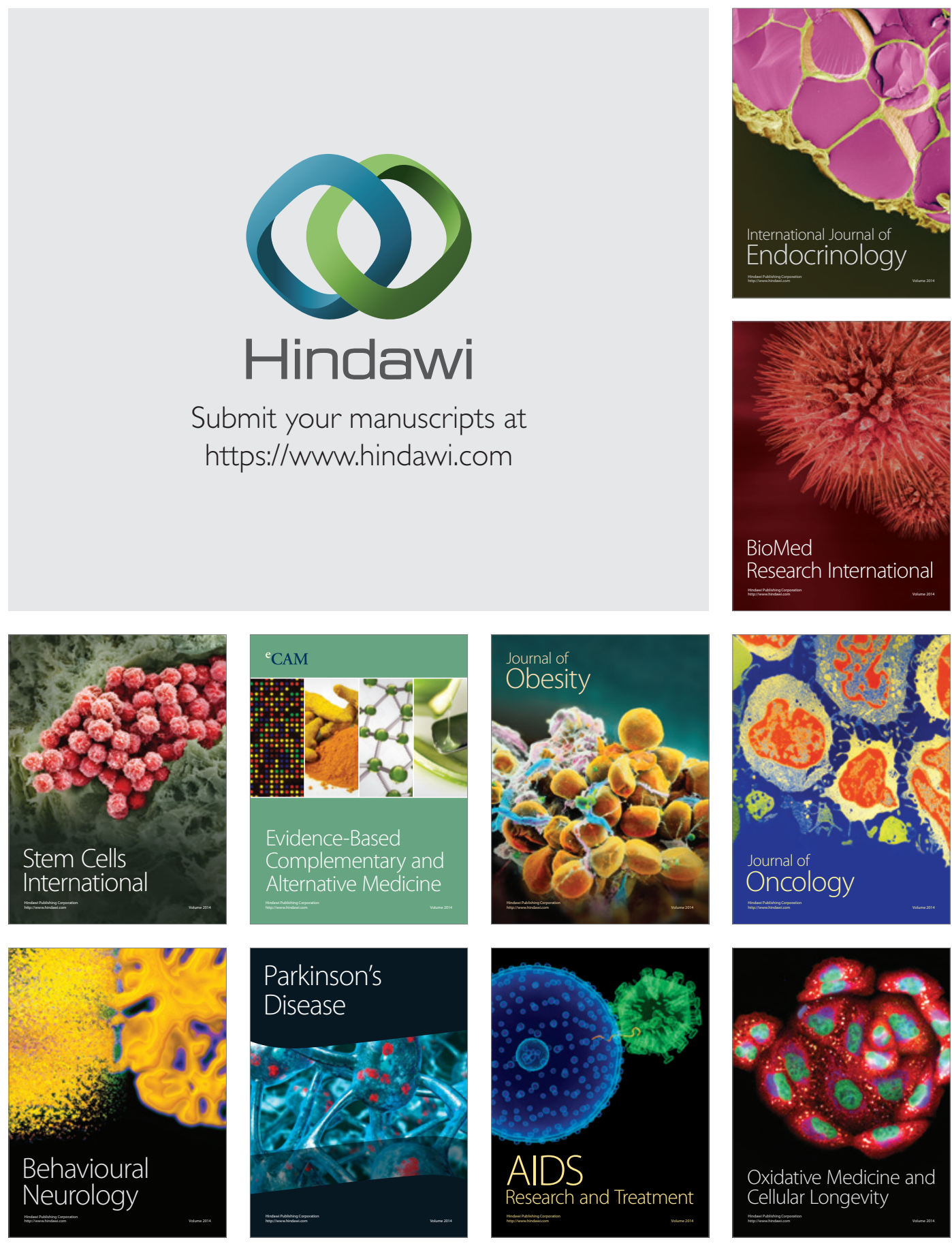